\title{
Qualidade do preenchimento da Caderneta de Saúde da Criança e fatores associados
}

\section{Quality of data on the Child Health Record and related factors}

\author{
1 Faculdade de Medicina, \\ Universidade Federal de \\ Minas Gerais, Belo Horizonte, \\ Brasil. \\ 2 Instituto de Ciências Exatas, \\ Universidade Federal de \\ Minas Gerais, Belo Horizonte, \\ Brasil. \\ 3 Secretaria Municipal de \\ Saúde, Prefeitura de Belo \\ Horizonte, Belo Horizonte, \\ Brasil. \\ Correspondência \\ C. R. L. Alves \\ Departamento de Pediatria, \\ Faculdade de Medicina, \\ Universidade Federal de \\ Minas Gerais. \\ Rua Tavares Bastos 287 \\ Belo Horizonte, $M G$ \\ 30380-040, Brasil. \\ lindgren@medicina.ufmg.br
}

\begin{abstract}
The Child Health Record (CHR) is an essential document for children's health care activities. A cross-sectional study was performed with simple random sampling, aimed at analyzing factors related to quality of data completion on the CHR. Interviews and direct verification of 365 CHRs from 9 health districts in Belo Horizonte, Minas Gerais State, Brazil, were performed. A scoring system was created to classify the CHRs in terms of quality of data completion and to relate them to explanatory variables. Odds ratios were calculated by logistic regression. Completion of the 20 scoring items varied from $3.1 \%$ (use of iron supplements) to $99.7 \%$ (date of birth). Factors associated with worse scores were: children $>12$ months old $(O R=1.77)$, mothers with $\leq 6$ years of schooling $(O R=1.97)$, children not treated by general practitioners $(O R=3.18)$, and mothers who had not received explanations on the CHR while in the maternity ward $(O R=1.77)$. The results show poor use of the CHR and emphasize the need for on-going efforts to train health professionals and prepare health services for their important role in the promotion of children's health.
\end{abstract}

Child Health; Medical Records; Primary Health Care; Health Promotion
Claudia Regina Lindgren Alves 1

Laura Maria de Lima Belizário Facury Lasmar 1

Lúcia Maria Horta Figueiredo Goulart ${ }^{1}$

Cristina Gonçalves Alvim 1

Gustavo Vieira Rodrigues Maciel 1

Maria Regina de Almeida Viana ${ }^{1}$

Enrico Antônio Colosimo 2

Guilherme Augusto Alves do Carmo 1

Juliana Goulart Dias da Costa 1

Maria Elizabeth Neves Magalhães 1

Marislaine Lumena de Mendonça ${ }^{3}$

Mirtes Maria do Vale Beirão 1

Zeína Soares Moulin 1

\section{Introdução}

A atenção primária e as ações voltadas para a vigilância à saúde constituem a base da organização da atenção à saúde infantil. Na Agenda de Compromissos com a Saúde Integral da Criança e Redução da Mortalidade Infantil 1, o Ministério da Saúde propõe, como eixos da assistência, 13 linhas de cuidado que privilegiam ações como acompanhamento do crescimento e desenvolvimento, incentivo ao aleitamento materno e alimentação saudável, prevenção de distúrbios nutricionais, imunização e atenção às doenças prevalentes, saúde bucal, saúde mental, prevenção de acidentes e maus-tratos, além de ações específicas dirigidas à mulher e ao recém-nascido.

No desenvolvimento dessas ações, a Caderneta de Saúde da Criança (CSC) apresenta-se como instrumento essencial de vigilância, por ser o documento onde são registrados os dados e eventos mais significativos para a saúde infantil, por possibilitar o diálogo entre a família e os diversos profissionais que atendem a criança e especialmente por pertencer à criança e à família e com elas transitar pelos diferentes serviços e níveis de atenção demandados no exercício do cuidado com a saúde 2 .

Implantada pelo Ministério da Saúde, em 2005, em substituição ao Cartão da Criança, a CSC é destinada a todos os pequenos cidadãos nascidos em território brasileiro. Esse documento é entregue às famílias nas maternidades e fica sob a sua guarda, 
devendo acompanhar a criança sempre que esta for levada a qualquer serviço de saúde. Na CSC, são registrados dados de identificação da criança, da história obstétrica e neonatal, da alimentação, do crescimento e desenvolvimento, do uso de suplementação de ferro e vitamina A, da saúde bucal, auditiva e visual, das vacinações, além do registro das intercorrências clínicas. A CSC, na terceira versão desde 2007, contém ainda orientações para a promoção da saúde e prevenção de agravos como acidentes e violência doméstica 3 .

Os registros devem ser efetuados por todos os profissionais de saúde que assistem à criança. Embora todos os cenários da atenção à saúde devam se responsabilizar pela verificação e o preenchimento da CSC, é particularmente nas maternidades e nos serviços de atenção primária que o adequado manejo deste instrumento constitui-se em permanente desafio, por serem estes os locais onde grande parte das informações é gerada.

O registro correto e completo das informações, além do diálogo com a família sobre as anotações realizadas são requisitos básicos para que a CSC cumpra seu papel de instrumento de comunicação, educação, vigilância e promoção da saúde infantil. A adequada utilização da CSC pelos profissionais possibilita maior valorização e apropriação do instrumento pela família, favorecendo sua maior adesão e co-responsabilização pelas ações de vigilância. Por outro lado, a qualidade dos registros na CSC pode revelar o funcionamento dos serviços e o desempenho de seus profissionais 4 .

No Brasil, ainda não foram encontradas publicações que avaliam a qualidade do preenchimento da CSC e são poucas as que avaliam o seu antecessor, o Cartão da Criança. Embora raros, esses estudos apontam falhas consideráveis na utilização desses instrumentos.

Em Feira de Santana (Bahia), a análise do Cartão da Criança de 2.319 menores de um ano evidenciou a ausência do registro do Apgar em $72 \%$ deles, falhas no registro do peso em $34 \%$ e falhas no registro dos marcos de desenvolvimento em $78 \%{ }^{4}$. Em Pernambuco, um estudo mostrou que $13 \%$ de 1.669 crianças não tinham o registro do peso de nascimento no cartão ${ }^{5}$. Em Belém (Pará), pesquisa com 560 crianças mostrou a baixa proporção (12\%) de registro completo, no Cartão da Criança, dos dados relativos ao nascimento 6 . Em Teresópolis (Rio de Janeiro), pesquisa com 329 menores de um ano mostrou a ausência de registro de peso nos últimos três meses em $30 \%$ dos cartões, ainda que mais de $90 \%$ dessas crianças tivessem comparecido à consulta pediátrica nesse período 7. Mais recentemente, em Belo Horizonte (Minas Gerais), foi realizado um estudo envolvendo 797 CSC de crianças nascidas em
2005, que revelou a precariedade do preenchimento dos dados sobre gravidez, parto e recémnascido ${ }^{8}$.

Os fatores relacionados à baixa qualidade do preenchimento da CSC, todavia, não são conhecidos. Presume-se que a identificação desses fatores possa subsidiar reflexões, quiçá mudanças, que venham favorecer a saúde infantil. Com essa perspectiva foi realizado este estudo, cujo objetivo é analisar os fatores associados à qualidade do preenchimento da CSC em Belo Horizonte.

\section{Metodologia}

Trata-se de um estudo transversal, cuja seleção da amostra foi aleatória simples entre crianças acompanhadas no Sistema Único de Saúde (SUS) do Município de Belo Horizonte. Foram incluídas crianças nascidas em Belo Horizonte entre 1o de maio de 2005 e 31 de janeiro de 2006, período em circulou a versão da CSC distribuída no ano de 2005 pelo Ministério da Saúde. Para participarem da pesquisa, as crianças deveriam estar acompanhadas pela mãe ou pai no Dia Nacional de Multivacinação em agosto de 2006. Além disso, foram incluídas as crianças que faziam controle de saúde nos serviços do SUS. As crianças que não possuíam ou não trouxeram suas CSC foram excluídas do estudo.

Os dados foram coletados por meio de entrevista com um dos pais da criança. No questionário utilizado, constavam 64 perguntas fechadas, das quais cinco se referiam à identificação da entrevista, 18 eram dirigidas ao acompanhante, abordando características demográficas da mãe, condições básicas de saúde da criança e assistência recebida por ela. Quarenta e uma questões eram preenchidas mediante a verificação direta das cadernetas, sendo, por isso, indispensável que a criança estivesse portando sua CSC para ser incluída no estudo.

Os entrevistadores foram 50 voluntários acadêmicos de medicina da Universidade Federal de Minas Gerais (UFMG), supervisionados por sete professores do Departamento de Pediatria da Faculdade de Medicina da UFMG e uma pediatra da Secretaria Municipal de Saúde de Belo Horizonte (SMSA-BH). Todos os pesquisadores receberam treinamento específico para realizar este projeto. Foi feito um estudo-piloto em uma unidade básica de saúde (UBS) para adequação do instrumento e da metodologia de coleta de dados.

O cálculo do tamanho da amostra foi feito com base no número de nascidos vivos em Belo Horizonte no período de maio de 2005 a janeiro de 2006: 22.311 crianças (Ministério da 
Saúde; http://tabnet.datasus.gov.br/cgi/tabcgi. exe?sinasc/cnv/nvMG.def, acessado em 23/ Jun/2006). Considerando uma freqüência esperada de preenchimento correto de $65 \%$, erro aceitável de \pm 5 pontos percentuais e intervalo de 95\% de confiança (IC95\%), estimou-se uma amostra de 344 crianças 8 . Somando-se uma perda de 5 a $6 \%$, o número calculado de crianças na amostra foi de 365. Este número de crianças foi distribuído entre as nove regionais de saúde de Belo Horizonte com base na proporção de nascidos vivos em cada uma. Em cada regional, foram sorteadas duas UBS entre aquelas que aplicaram mais de 200 doses de vacinas em menores de um ano durante a campanha de vacinação de junho de 2005 (dados fornecidos pela SMSA-BH), asse- gurando que o número mínimo de crianças calculado na amostra fosse alcançado. As equipes de pesquisadores se distribuíram, portanto, por 18 das 142 UBS do sistema municipal de saúde ${ }^{9}$, como mostra a Figura 1. Foi feita seleção sistemática das crianças (amostra aleatória simples). Foram entrevistadas as crianças elegíveis de número ímpar pela ordem de chegada em cada UBS, durante todo o horário de funcionamento do posto de vacinação no dia da campanha.

Foi criado um sistema de escore para classificar as CSC de acordo com a qualidade do seu preenchimento a exemplo da metodologia utilizada em estudos abordando outros temas 10,11. Essa classificação se baseou na avaliação do preenchimento de 20 variáveis entre os mais de 50

Figura 1

Mapa do Município de Belo Horizonte, Minas Gerais, Brasil, dividido por regionais de saúde com todas as suas unidades básicas de saúde (UBS) e, em destaque, a localização das UBS sorteadas para este estudo.

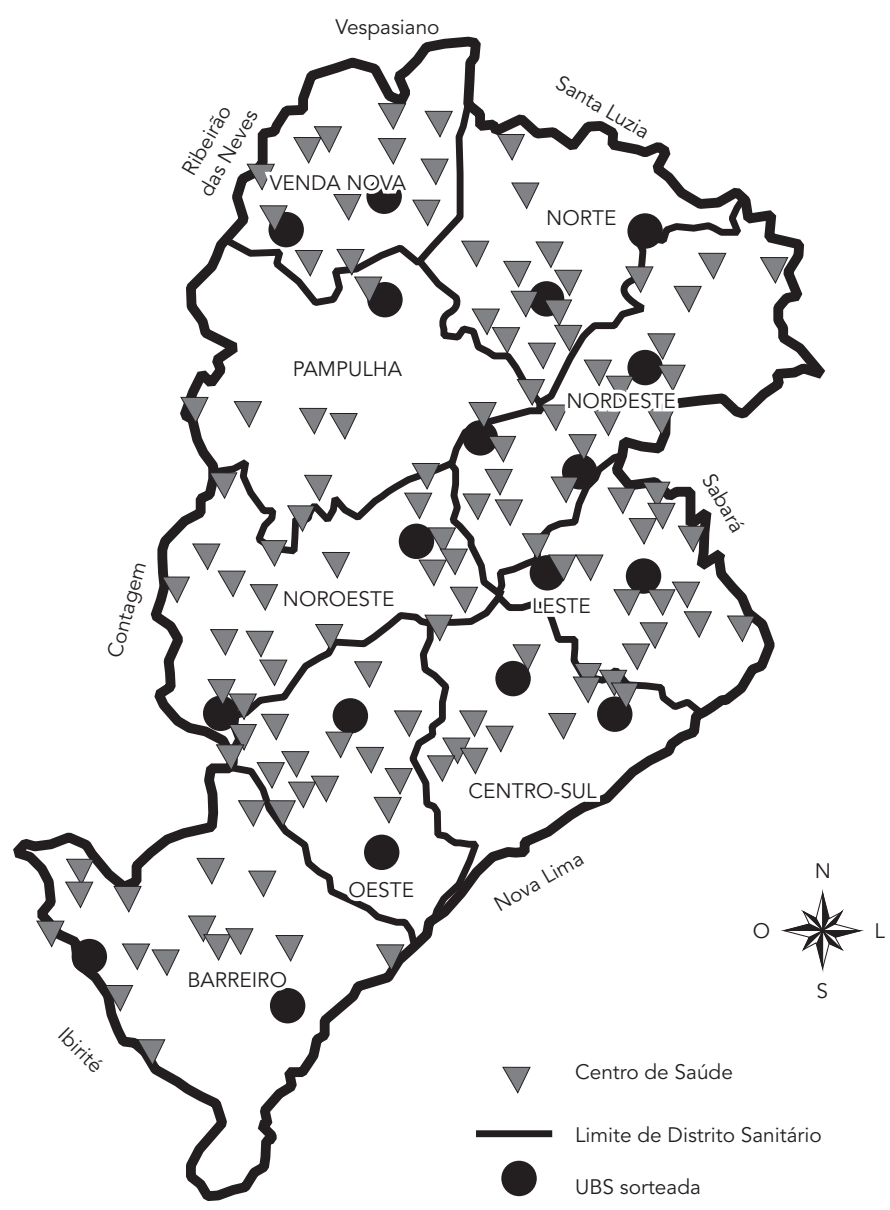


itens disponíveis na versão 2005 da CSC. Com base na prática clínica de um grupo de professores do Departamento de Pediatria da Faculdade de Medicina da UFMG, com vasta experiência em atenção primária, foram selecionados os itens da CSC essenciais ao acompanhamento da saúde da criança e cujo preenchimento foi considerado como o mínimo indispensável. Optou-se por consolidar todas as informações em uma única variável que refletisse a qualidade do preenchimento de cada CSC e relacioná-la aos possíveis fatores associados ao melhor ou pior preenchimento.

A avaliação do preenchimento da CSC tomou como referência as orientações do Manual para Utilização da Caderneta de Saúde da Criança ${ }^{2}$. $\mathrm{O}$ item foi considerado preenchido corretamente quando estava de acordo com as orientações do Ministério da Saúde. Quando não havia normalização sobre o assunto, considerou-se apenas preenchido ou não preenchido. Atribuiu-se o valor 1 para itens preenchidos corretamente e o valor zero para os itens preenchidos incorretamente ou não preenchidos. O intervalo máximo admitido para registro do peso e do perímetro cefálico nos gráficos da CSC foi três meses, de acordo com o calendário mínimo de acompanhamento da saúde da criança do Ministério da Saúde na faixa etária estudada 1 . A nota atribuída ao item "desenvolvimento neuropsicomotor" foi uma composição do preenchimento das habilidades esperadas até a idade cronológica da criança. Como todas as crianças tinham no mínimo sete meses de idade, esperava-se que tivessem pelo menos três registros de avaliação do desenvolvimento na CSC. A pontuação final recebida por cada CSC foi dada pelo somatório dos valores recebidos em cada item, variando de 0 a 20 pontos. Quanto maior o valor do escore, mais itens estavam preenchidos segundo os critérios adotados neste estudo. A veracidade dos registros não foi investigada.

A variável resposta estudada foi o escore de preenchimento da CSC, transformado em variável binária segundo o percentual de preenchimento dos itens incluídos no sistema de escore: $60 \%$ ou menos dos itens preenchidos $(\leq 12$ pontos) e mais de $60 \%$ dos itens preenchidos (> 12 pontos). Este ponto de corte foi definido com base na distribuição estatística dos escores encontrados, uma vez que não foram encontradas referências na literatura que pudessem nortear o ponto de corte. Na análise univariada, a associação entre as variáveis independentes e o escore foi avaliada pelo cálculo do odds ratio (OR) e seu respectivo IC95\%, pelos testes do quiquadrado de Pearson e exato de Fisher. Em uma segunda etapa, as variáveis associadas ao escore $\leq 12$ pontos foram avaliadas pelo cálculo do OR obtido pelo ajuste de modelos de regressão logística. O modelo inicial da análise multivariada incluiu todas as variáveis da análise univariada cujos valores de $\mathrm{p}$ foram menores que 0,20 12. A exclusão de cada variável foi feita pelo teste de Wald e os modelos foram comparados utilizando-se o teste da razão de verossimilhança. A adequação do modelo final foi assegurada pelo teste de Hosmer-Lemeshow. O nível de significância de cada variável do modelo final foi fixado em 0,05.

O cálculo do tamanho da amostra, o banco de dados e a análise univariada foram feitos no programa Epi Info versão 6.04 (Centers for Disease Control and Prevention, Atlanta, Estados Unidos) e a análise multivariada no pacote estatístico MULTLR.

O projeto de pesquisa, o instrumento de coleta de dados e o Termo de Consentimento Livre e Esclarecido foram aprovados pela Câmara do Departamento de Pediatria da Faculdade de Medicina da UFMG e pelo Comitê de Ética em Pesquisa da SMSA-BH. As mães entrevistadas foram esclarecidas sobre os objetivos e métodos da pesquisa e assinaram livremente o termo de consentimento.

\section{Resultados}

Compareceram às UBS sorteadas, no dia da campanha de vacinação, 728 crianças elegíveis para o estudo. Destas, dez foram sorteadas e recusaram-se a participar da pesquisa. Em todas as regionais, exceto uma, o número mínimo de crianças calculado foi atendido. As perdas corresponderam a 11 crianças cujos questionários não estavam completos, em variáveis que impossibilitavam o cálculo do escore de preenchimento. Foram analisados 355 questionários.

A Tabela 1 mostra as características gerais da amostra estudada. Em $84 \%$ dos casos a entrevista foi realizada com a mãe da criança. A idade das mães variou entre 15 e 46 anos, com mediana de 26 anos. Cerca de 15\% das mães eram adolescentes. A mediana de anos de estudo das mães foi nove anos, e apenas $2 \%$ delas tinham nível superior completo ou incompleto.

A idade das crianças variou entre 6,8 e 15,8 meses (mediana de 11 meses) e 48,4\% delas eram do sexo feminino. De acordo com o registro nas CSC, o peso ao nascer variou de 735 a 4.285g, sendo a mediana de 3.110 g. Cerca de $10 \%$ das crianças pesavam menos de $2.500 \mathrm{~g}$ ao nascer e $7 \%$ delas nasceram com menos de 37 semanas de idade gestacional. O percentual de CSC sem informação sobre a idade gestacional foi de quase $17 \%$ (60 casos). 
Características gerais da amostra estudada. Belo Horizonte, Minas Gerais, Brasil.

\begin{tabular}{|c|c|c|}
\hline Variável [Mediana/Amplitude] & $\mathrm{n}$ & $\%$ \\
\hline \multicolumn{3}{|c|}{ Idade das mães (anos) [26 anos/15-46 anos] } \\
\hline$\leq 19$ & 54 & 15,2 \\
\hline$>19$ & 298 & 84,0 \\
\hline Sem informação & 3 & 0,8 \\
\hline \multicolumn{3}{|c|}{ Anos de estudo das mães [9 anos/0-20 anos] } \\
\hline$\leq 6$ & 81 & 22,8 \\
\hline$>6$ & 272 & 76,6 \\
\hline Sem informação & 2 & 0,6 \\
\hline \multicolumn{3}{|c|}{ Idade das crianças (meses) [11 meses/6,8-15,8 meses] } \\
\hline$\leq 12,0$ & 229 & 64,5 \\
\hline$>12,0$ & 126 & 35,5 \\
\hline \multicolumn{3}{|l|}{ Sexo das crianças } \\
\hline Masculino & 183 & 51,6 \\
\hline Feminino & 172 & 48,4 \\
\hline \multicolumn{3}{|c|}{ Peso ao nascer (gramas) [3.110 gramas/735-4.285 gramas] } \\
\hline$<2.500$ & 35 & 9,9 \\
\hline$\geq 2.500$ & 309 & 87,0 \\
\hline Sem informação & 11 & 3,1 \\
\hline \multicolumn{3}{|c|}{ Idade gestacional (semanas) [39 semanas/27-42 semanas] } \\
\hline$<37$ & 26 & 7,3 \\
\hline$\geq 37$ & 269 & 75,8 \\
\hline Sem informação & 60 & 16,9 \\
\hline \multicolumn{3}{|l|}{ Acompanhamento da criança } \\
\hline Unidades básicas de saúde & 332 & 93,5 \\
\hline Hospitais & 16 & 4,5 \\
\hline Sem informação & 7 & 2,0 \\
\hline \multicolumn{3}{|c|}{ Profissionais envolvidos no acompanhamento da criança * } \\
\hline Pediatra & 288 & 81,1 \\
\hline Enfermeiro & 115 & 32,3 \\
\hline Médico generalista & 50 & 14,0 \\
\hline Médico especialista & 7 & 1,9 \\
\hline Sem informação & 15 & 4,1 \\
\hline \multicolumn{3}{|c|}{ Explicações sobre a CSC na maternidade } \\
\hline Sim & 177 & 49,9 \\
\hline Não & 151 & 42,5 \\
\hline Sem informação & 27 & 7,6 \\
\hline \multicolumn{3}{|c|}{ Explicações sobre o gráfico peso X idade da CSC } \\
\hline Sim & 200 & 56,3 \\
\hline Não & 138 & 38,9 \\
\hline Sem informação & 17 & 4,8 \\
\hline \multicolumn{3}{|c|}{ Anotações feitas pelas mães na CSC } \\
\hline Sim & 77 & 21,7 \\
\hline Não & 271 & 76,3 \\
\hline Sem informação & 7 & 2,0 \\
\hline \multicolumn{3}{|c|}{ Escore (pontos) [14 pontos/4-19 pontos] } \\
\hline$\leq 12$ & 113 & 31,8 \\
\hline$>12$ & 242 & 68,2 \\
\hline Total & 355 & 100,0 \\
\hline
\end{tabular}

CSC: Caderneta de Saúde da Criança.

* Podia ser marcada mais de uma opção. Percentual calculado para o total de entrevistas realizadas. 
Aproximadamente 93\% das crianças eram regularmente acompanhadas em UBS. As demais crianças eram acompanhadas em hospitais ou eram levadas aos serviços de pronto-atendimento, quando necessário. Sete entrevistadas não souberam informar o tipo de serviço do SUS onde seus filhos eram acompanhados.

A pergunta sobre quais profissionais estavam envolvidos no acompanhamento da criança permitia que a mãe escolhesse mais de uma opção. Foi verificado que existem várias composições de profissionais envolvidos no acompanhamento da criança em Belo Horizonte. O pediatra participava deste acompanhamento em mais de $80 \%$ dos casos, sendo o único citado por $54 \%$ dos responsáveis. O médico generalista foi citado como um dos responsáveis pelo acompanhamento da criança em $14 \%$ das entrevistas, sendo o único citado em 7\%. Na Tabela 1 também são apresentados os percentuais de crianças acompanhadas por enfermeiros e médicos especialistas.

Metade das mães entrevistadas não recebeu qualquer informação sobre a CSC durante a per- manência na maternidade e 38,9\% delas nunca receberam informações sobre o gráfico peso $\mathrm{X}$ idade disponível na caderneta. As mães relataram ter feito anotações na CSC de seus filhos em $21,7 \%$ das entrevistas.

Os itens escolhidos para compor o escore de preenchimento e o percentual de preenchimento correto em cada um deles são apresentados na Tabela 2. O escore de preenchimento da CSC variou entre 4 e 19 pontos, sendo a média igual a 14 pontos. Cerca de $30 \%$ das CSC obtiveram escore menor ou igual a 12, considerado insatisfatório.

Verificou-se que o preenchimento correto dos itens analisados foi bastante heterogêneo, variando de $3,1 \%$ (registro do uso de ferruginoso) a 99,7\% (data de nascimento). Chama atenção o baixo percentual de cadernetas em que os gráficos disponíveis foram utilizados adequadamente. O peso ao nascer estava registrado corretamente em $94,1 \%$ das CSC, embora apenas em $69,3 \%$ delas este dado estivesse registrado no gráfico apropriado. O perímetro cefálico ao nascer estava registrado no gráfico em $15,5 \%$ das CSC,

Tabela 2

Composição do escore e percentual de preenchimento correto em cada item. Belo Horizonte, Minas Gerais, Brasil.

\begin{tabular}{|c|c|}
\hline Itens da CSC & $\begin{array}{c}\text { Preenchimento } \\
\text { correto (\%) }\end{array}$ \\
\hline 1. Nome da criança & 93,8 \\
\hline 2. Data de nascimento & 99,7 \\
\hline 3. Nome da mãe & 98,9 \\
\hline 4. Peso ao nascer & 94,1 \\
\hline 5. Comprimento ao nascer & 91,2 \\
\hline 6. Perímetro cefálico ao nascer & 85,6 \\
\hline 7. Apgar no 5 o minuto & 53,5 \\
\hline 8. Tipo de parto & 89,3 \\
\hline 9. Orientação para levar o recém-nascido à unidade básica de saúde na primeira semana de vida & 33,0 \\
\hline 10. Trimestre de início do pré-natal & 58,0 \\
\hline 11. Número de consultas de pré-natal & 69,9 \\
\hline 12. Idade gestacional da criança & 72,4 \\
\hline 13. Tipo de alimentação da criança na alta da maternidade & 67,3 \\
\hline 14. Perímetro cefálico ao nascer marcado no gráfico & 15,5 \\
\hline 15. Idade em que o último ponto de perímetro cefálico foi marcado no gráfico * & 30,7 \\
\hline 16. Peso ao nascer marcado no gráfico & 69,3 \\
\hline 17. Idade em que o último ponto de peso foi marcado no gráfico * & 59,4 \\
\hline 18. Anotação sobre o desenvolvimento neuropsicomotor ** & 18,9 \\
\hline 19. Registro do uso de ferruginoso & 3,1 \\
\hline 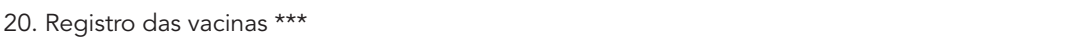 & 94,6 \\
\hline
\end{tabular}

* Diferença entre idade marcada e idade cronológica $\leq 3$ meses $=1$ e $>3$ meses $=0$;

** Faixas etárias disponíveis na Caderneta de Saúde da Criança: 1-2 meses, 3-4 meses, 5-6 meses, 7-9 meses, 10-12 meses e

13-18 meses. Anotação em três ou mais faixas etárias = 1 e em menos de três faixas etárias = 0 ;

*** Calendário completo para a idade ou vacinas com menos de um mês de atraso $=1$ e vacinas com um mês ou mais de atraso $=0$. 
embora 85,6\% delas tivessem essa informação disponível nas primeiras páginas da caderneta.

Em relação ao uso de ferro profilático, verificou-se o registro em $3,1 \%$ das CSC, mesmo que tenha sido informado pelos acompanhantes que $65 \%$ das crianças estavam em uso de suplementação de ferro por ocasião do estudo.

Cerca de $95 \%$ das CSC estavam com o calendário vacinal em dia (informação obtida antes de a criança ser vacinada na campanha).

Na Tabela 3, são apresentadas as variáveis explicativas incluídas na análise univariada e os resultados do cruzamento dessas variáveis com o escore de preenchimento das CSC. As variáveis com valor de $\mathrm{p} \leq 0,20$ (em negrito na Tabela 3) foram incluídas no modelo inicial da análise multivariada. A variável idade gestacional, apesar de ter apresentado valor de $p=0,15$, não foi incluída na análise multivariada por apresentar um grande número de CSC sem tal informação (60 casos).

Para a análise, a variável "profissionais envolvidos no acompanhamento da saúde da criança” foi desdobrada em outras quatro. Uma variável

Tabela 3

Variáveis explicativas incluídas na análise univariada e sua relação com a pontuação recebida pela Caderneta de Saúde da Criança (CSC) no escore de preenchimento. Belo Horizonte, Minas Gerais, Brasil.

\begin{tabular}{|c|c|c|c|c|}
\hline \multirow[t]{2}{*}{ Variáveis explicativas } & \multicolumn{2}{|c|}{ Escore (pontos) } & \multirow[t]{2}{*}{ Valor de $p$ * } & \multirow[t]{2}{*}{ OR (IC95\%) } \\
\hline & $\leq 12$ & $>12$ & & \\
\hline \multicolumn{5}{|l|}{ Idade das mães (anos) } \\
\hline$\leq 19$ & 17 & 37 & 0,95 & $0,98(0,50-1,92)$ \\
\hline$>19$ & 95 & 203 & & \\
\hline \multicolumn{5}{|l|}{ Anos de estudo das mães } \\
\hline$\leq 6$ & 33 & 48 & 0,06 & $1,65(0,95-2,86)$ \\
\hline$>6$ & 80 & 192 & & \\
\hline \multicolumn{5}{|l|}{ Idade das crianças (meses) } \\
\hline$\leq 12,0$ & 63 & 166 & 0,03 & $1,73(1,06-2,83)$ \\
\hline$>12,0$ & 50 & 76 & & \\
\hline \multicolumn{5}{|l|}{ Sexo das crianças } \\
\hline Masculino & 125 & 58 & 0,95 & $0,99(0,61-1,59)$ \\
\hline Feminino & 117 & 55 & & \\
\hline \multicolumn{5}{|l|}{ Peso ao nascer (gramas) } \\
\hline$<2.500$ & 10 & 25 & 0,85 & $0,93(0,40-2,14)$ \\
\hline$\geq 2.500$ & 93 & 216 & & \\
\hline \multicolumn{5}{|l|}{ Idade gestacional (semanas) ** } \\
\hline$<37$ & 8 & 18 & 0,15 & $1,90(0,71-4,99)$ \\
\hline$\geq 37$ & 51 & 218 & & \\
\hline \multicolumn{5}{|l|}{ Acompanhamento da criança } \\
\hline Unidades Básicas de saúde & 102 & 230 & 0,11 & $2,25(0,74-6,89)$ \\
\hline Hospitais & 8 & 8 & & \\
\hline \multicolumn{5}{|c|}{ Participação do generalista na assistência } \\
\hline Sim & 9 & 41 & 0,02 & $2,40(1,06-5,58)$ \\
\hline Não & 100 & 190 & & \\
\hline \multicolumn{5}{|c|}{ Explicações sobre a CSC na maternidade } \\
\hline Sim & 43 & 134 & 0,01 & $1,89(1,14-3,14)$ \\
\hline Não & 57 & 94 & & \\
\hline \multicolumn{5}{|c|}{ Explicações sobre o gráfico peso X idade } \\
\hline Sim & 54 & 146 & 0,04 & $1,63(1,00-2,69)$ \\
\hline Não & 52 & 86 & & \\
\hline \multicolumn{5}{|c|}{ Anotações feitas pelas mães na CSC } \\
\hline Sim & 19 & 58 & 0,15 & $1,52(0,82-2,83)$ \\
\hline Não & 90 & 181 & & \\
\hline
\end{tabular}

* Em negrito, as variáveis incluídas no modelo inicial da análise multivariada (regressão logística);

** Não incluída na análise multivariada. 
comparando as categorias de profissionais entre si (pediatras, generalistas, enfermeiros e outras), cujo valor de p foi 0,87 ; outra comparando crianças com e sem acompanhamento por pediatras ( $p=0,25)$, uma terceira comparando crianças com e sem acompanhamento por enfermeiros $(p=0,83)$ e, por último, crianças com e sem acompanhamento por médicos generalistas $(p=0,02)$. Apenas esta última variável foi apresentada na Tabela 3 e incluída no modelo inicial da análise multivariada.

O modelo final da análise multivariada (regressão logística) está descrito na Tabela 4. As interações entre as variáveis foram testadas e não houve significância estatística.

Tabela 4

Fatores associados ao escore $\leq 12$ pontos no modelo final da análise multivariada. Belo Horizonte, Minas Gerais, Brasil.

\begin{tabular}{lcc}
\hline Variáveis & Valor de p & OR (IC95\%) \\
\hline Crianças com mais de 12 meses de idade & 0,03 & $1,77(1,07-2,94)$ \\
Mães com seis anos ou menos de estudo & 0,02 & $1,97(1,10-3,50)$ \\
Crianças não acompanhadas por & 0,01 & $3,18(1,35-7,53)$ \\
médicos generalistas & 0,02 & $1,77(1,08-2,91)$ \\
Mães que não receberam explicações & & \\
sobre a CSC na maternidade & & \\
\hline
\end{tabular}

CSC: Caderneta de Saúde da Criança.

Teste de Hosmer-Lemeshow = 0,708 (ajuste do modelo).

\section{Discussão}

A realização de inquéritos nos dias de campanha de vacinação vem se tornando uma prática comum entre os pesquisadores, especialmente aqueles ligados à saúde pública 13 . As altas taxas de comparecimento de crianças menores de cinco anos aos postos de vacinação em dias de campanhas e o baixo custo operacional oferecem excelentes oportunidades de obtenção de amostras representativas. Por mais que os critérios de inclusão adotados no presente estudo não permitissem a obtenção de uma amostra representativa da população infantil de Belo Horizonte, acredita-se que o processo de aleatorização das UBS e das crianças entrevistadas possa refletir a realidade da população acompanhada no SUS municipal nesta faixa etária. Atualmente, perto de $76 \%$ da população do município é assistida pelo Programa Saúde da Família nas UBS 9. A proporção de crianças nas regionais foi, em ge- ral, respeitada e o percentual de perdas foi considerado aceitável (3\%). A possibilidade de viés de informação e memória foi reduzida pelo fato da principal informante ter sido a mãe na maior parte das entrevistas e as crianças terem menos de 16 meses.

Diferentemente da maior parte dos estudos sobre o uso do Cartão da Criança no Brasil, que se limita a descrever os percentuais de preenchimento de itens isolados, este estudo apresenta dois aspectos relevantes. Primeiro, foi elaborado um sistema de escore para avaliação da qualidade do preenchimento da CSC, permitindo a análise de um conjunto de dados relevantes para a saúde da criança. Tal escore pode, em futuros estudos, possibilitar comparações com outras regiões, ao longo do tempo e após intervenções que visem promover a utilização da CSC. O outro ganho foi a possibilidade de estudar os fatores associados ao melhor ou pior preenchimento desse instrumento. Atendendo a este objetivo, foi escolhido um conjunto amplo de variáveis sócio-demográficas, biológicas e assistenciais que pudessem influenciar o preenchimento da CSC.

Apesar de a CSC ter sido implementada recentemente, os 20 itens selecionados para a composição do escore refletem as ações básicas propostas para o acompanhamento da saúde da criança há décadas no Brasil. O escore médio encontrado correspondeu a $70 \%$ de preenchimento dos itens selecionados e nenhuma CSC recebeu pontuação máxima. Considerando que o escore foi composto por itens considerados essenciais, estes resultados sugerem uma utilização ainda precária da CSC na rede pública de saúde de Belo Horizonte. Outros estudos realizados no Brasil já levantavam o problema em relação ao Cartão da Criança que precedeu a CSC 4,5,6,7,15,16.

O uso adequado de instrumentos para acompanhamento do crescimento infantil representa um desafio para muitos países do mundo. Estudo realizado entre 1998 e 2002 pela Organização Mundial da Saúde envolvendo 178 países mostrou que, em $80 \%$ deles, os profissionais de saúde encontravam dificuldades no uso dos cartões para acompanhamento do crescimento infantil. Os problemas encontrados eram de natureza conceitual e operacional e variavam desde a compreensão das curvas de crescimento até a ausência de equipamentos adequados para pesar e medir as crianças 16 .

Os melhores percentuais de preenchimento foram nos itens relativos à identificação da criança, dados do nascimento (tipo de parto, peso, comprimento, perímetro cefálico) e registro de vacinas. Com exceção do Apgar no 5o minuto, todas essas informações estavam preenchidas adequadamente em mais de 85\% das CSC analisadas. 
É interessante notar que houve melhora do registro dessas informações quando os resultados são comparados aos do estudo realizado em 2005 em Belo Horizonte ${ }^{8}$. Apesar dos avanços, os percentuais de preenchimento de itens importantes como o pré-natal, a idade gestacional, o tipo de alimentação na alta da maternidade foram ainda abaixo do esperado (58 a $72 \%$ ).

O campo destinado à orientação para levar o recém-nascido à UBS na primeira semana de vida para receber as ações da "Primeira Semana de Saúde Integral”, que inclui, entre outras, a triagem neonatal, a avaliação da amamentação e do puerpério e a imunização contra hepatite e tuberculose, estava preenchido em apenas um terço das CSC. A realização dessas ações representa uma importante iniciativa para a redução da mortalidade infantil e materna e é fundamental que a mãe seja orientada ainda na maternidade 1,17 . O baixo índice de registro dessa orientação na CSC sugere uma descontinuidade entre as ações iniciadas na maternidade e as propostas para a atenção básica.

A CSC trouxe consigo não só mudanças na concepção e na forma do instrumento como introduziu também mudanças operacionais que não podem ser desprezadas 8. A partir de 2005, os hospitais e maternidades se tornaram os responsáveis pela distribuição e pelo registro das informações relativas à gravidez, parto e período neonatal na CSC. Mudanças, com freqüência, exigem tempo e sensibilização para serem incorporadas na rotina de funcionamento dos serviços. Isso, talvez, explique o pequeno número de mães que recebeu orientações sobre a CSC nas maternidades neste estudo. Em pesquisa realizada em Belo Horizonte, em 2005 8, os autores encontraram associação significativa $(\mathrm{p}=0,001)$ entre o fato de a mãe não ter recebido informações sobre a CSC na maternidade e o não-reconhecimento pela mãe das funções da CSC para o acompanhamento da saúde da criança.

Os percentuais de preenchimento das curvas de crescimento do perímetro cefálico (PC) e peso foram considerados insatisfatórios. O Ministério da Saúde recomenda que, na faixa etária estudada, a criança deve ser atendida pelo menos a cada três meses pela equipe de saúde. Entretanto, constatou-se que apenas $15,5 \%$ e 59,4\% das crianças tiveram suas medidas registradas nos gráficos de PC e peso X idade, respectivamente, nos três meses que antecederam à entrevista. $\mathrm{O}$ registro do peso no gráfico neste estudo foi superior ao encontrado em Feira de Santana $(41,1 \%)$ e inferior àqueles observados em Teresópolis $(70,4 \%)$ e Montes Claros (Minas Gerais; 72,7\%) 4,7,14.

Ressalta-se a discrepância entre os registros de PC e de peso. A CSC de 2005 foi o primeiro instrumento a incorporar o gráfico PC X idade. É preciso questionar se a medida do PC é, de fato, feita rotineiramente. Há anos, os profissionais que atuam na saúde pública vêm concentrando seus esforços no combate à desnutrição em detrimento das demais ações.

Apenas 18,9\% das CSC tinham pelo menos três anotações sobre desenvolvimento neuropsicomotor (DNPM). Outros autores têm chamado a atenção para a necessidade de se valorizar mais efetivamente a avaliação do DNPM. Em Belém, estudo com profissionais que atuam na atenção básica mostrou que cerca de $70 \%$ deles faziam avaliação rotineira do DNPM, mas apenas 32\% deles utilizavam algum instrumento para isto. $\mathrm{O}$ Cartão da Criança foi o principal instrumento citado por estes profissionais ${ }^{18}$. Apesar disto, em Feira de Santana evidenciou-se que menos de $8 \%$ de 2.190 crianças tinham os registros de desenvolvimento completos no Cartão da Criança 4. Por outro lado, o registro de marcos do DNPM na caderneta francesa variou de 74 a 93\%, dependendo da idade da criança, o que mostra uma nítida diferença cultural em relação aos aspectos da saúde da criança considerados relevantes 19 .

Cerca de 95\% das crianças pesquisadas estavam com as vacinas em dia e devidamente registradas na CSC. Se por um lado, esse dado atesta o contato freqüente e regular das crianças com os serviços de saúde e dos profissionais de saúde com a CSC; por outro, reflete uma concepção da CSC como o "cartão de vacina", historicamente construída. A utilização do instrumento para acompanhamento de outros itens importantes na promoção da saúde da criança não encontra a mesma adesão. Semelhante ao que foi observado quanto às anotações sobre DNPM, as informações sobre o uso de ferro profilático estavam registradas em apenas em 3,1\% das cadernetas, embora $65 \%$ dos acompanhantes relatassem seu uso.

A utilização do sistema de escores permitiu identificar quatro grupos de crianças com risco de terem suas CSC mais mal preenchidas: aquelas cujas mães tinham seis anos ou menos de estudo; aquelas com idade superior a 12 meses; aquelas cujas mães não receberam explicações sobre a CSC na maternidade e as crianças não acompanhadas por um médico generalista.

A baixa escolaridade materna é freqüentemente associada a riscos para a saúde da criança. O estudo de Feira de Santana evidenciou associação positiva entre a escolaridade materna e a freqüência de registro do DNPM, mas essa associação não foi verificada para o preenchimento da curva de crescimento ${ }^{4}$. Deve-se ter cuidado na interpretação deste dado, pois a responsabilidade pela CSC deve ser compartilhada entre a 
família e a equipe de saúde. Este resultado pode, por um lado, sugerir que as mães menos instruídas têm maior dificuldade em assumir seu papel em relação à CSC. Mas, por outro lado, indica a necessidade de repensar a organização da assistência nas equipes de saúde, de modo a valorizar a CSC como instrumento de vigilância da saúde da criança, especialmente nos grupos com maior vulnerabilidade social.

As consultas de puericultura tendem a ser mais freqüentes no primeiro ano de vida, fato que pode permitir melhor utilização da CSC. Após esta idade, as mães tendem a consultar quando existe uma queixa e a CSC pode não ser tão valorizada. Mas, além disso, as crianças mais velhas receberam suas CSC logo após o seu lançamento pelo Ministério da Saúde. Apesar da ampla divulgação na imprensa leiga, não houve qualquer capacitação ou sensibilização dos profissionais de saúde para a utilização do novo instrumento. No entanto, entre 2005 e 2006, muitos profissionais da rede básica de saúde do Município de Belo Horizonte, que cursavam a especialização em saúde da família na UFMG, foram fortemente motivados a utilizar a CSC como instrumento de acompanhamento da saúde integral da criança. Estudo avaliando os conhecimentos dos médicos matriculados nesse mesmo curso entre 2002 e 2003 demonstrou aumento significativo dos percentuais de acerto nos testes aplicados antes e depois do módulo de saúde da criança, sobretudo em temas relacionados às ações básicas 20 . É possível que, após esta sensibilização, os profissionais tenham se tornado mais empenhados no uso e no registro das informações na CSC das crianças mais jovens.

Ficou demonstrada também a importância da atuação dos profissionais na maternidade quanto a orientações a respeito da CSC. O risco de a CSC das crianças cujas mães não receberam orientações na maternidade estarem mais mal preenchidas foi 1,77 vez o risco daquelas orientadas. Possivelmente, as mães orientadas estiveram mais empenhadas em levar a CSC aos atendimentos de seus filhos e, talvez, tenham sido mais exigentes quanto à sua utilização pelos profissionais. Também é admissível que essas crianças tenham nascido em maternidades já mais comprometidas com o uso deste instrumento, levando a um melhor preenchimento dos itens sob sua responsabilidade. A co-responsabilização de famílias, profissionais e serviços é a chave para o bom uso da CSC.

Por fim, crianças acompanhadas por equipes sem a participação de médicos generalistas tiveram um risco três vezes maior de terem suas CSC mais mal preenchidas do que as demais. Embora muitas vezes seja difícil para as mães identificarem a formação dos profissionais que assistem seus filhos, particularmente quando isto é feito por uma equipe multiprofissional, este resultado é compatível com os encontrados em outros estudos que compararam o desempenho das equipes de saúde família com as equipes que adotam o modelo tradicional de assistência. Alguns estudos recentes vêm mostrando maior sensibilidade dos profissionais do PSF para as ações básicas de saúde da criança, como o acompanhamento do crescimento e do desenvolvimento infantil e a promoção do aleitamento materno 15,21,22.

Desde 2004, está em vigor, em Belo Horizonte, a Agenda de Compromissos com a Saúde Integral da Criança e Redução da Mortalidade Infantil 1, que traz orientações para as ações dos profissionais que lidam com a criança na atenção primária. Na Agenda, está previsto um calendário mínimo de acompanhamento da saúde da criança, que envolve uma equipe multiprofissional composta por agentes comunitários de saúde, enfermeiros, pediatras e médicos de família, cujas ações podem ser de caráter individual ou coletivo. Na amostra estudada, o pediatra aparece como principal responsável por este acompanhamento, mas apenas 3,6\% das mães citaram a participação da equipe completa - médico de família, pediatra e enfermeiro - no acompanhamento de seus filhos como proposto na Agenda. A tradição de acompanhamento da saúde da criança por pediatras, a alta rotatividade de médicos nas equipes de saúde da família no município e o fato de muitos antigos pediatras da rede municipal terem se tornado médicos de família após a implantação do programa em 2002 podem explicar tais resultados.

Surpreende o número significativo de pais entrevistados que assumiram já ter feito alguma anotação na CSC de seus filhos $(21,7 \%)$. Um estudo da Sociedade Francesa de Pediatria, publicado em 2003, registrou que apenas 13 dos 1.685 pais entrevistados haviam feito anotações nas cadernetas. Os autores consideraram pequena a participação dos pais e questionaram se, apesar dos mais de 50 anos de utilização naquele país, a CSC seria entendida como um documento exclusivamente médico ${ }^{19}$. Ainda que a participação dos pais possa sinalizar um maior envolvimento deles com o instrumento e deva ser encorajado, no presente estudo não se verificou associação desta variável com melhores escores de preenchimento da CSC $(p=0,15)$.

Ao realizar este estudo, os autores tinham em mente poder alertar os gestores do sistema de saúde sobre a necessidade de sensibilizar e capacitar seus profissionais para um melhor uso da CSC e de estimular uma melhor organização 
dos serviços públicos de saúde no município. Neste sentido, acredita-se que os resultados encontrados serão capazes de alcançar este propósito. Todavia, novos estudos incluindo também crianças de outras faixas etárias e aquelas assistidas pela rede privada de saúde, bem como a análise de outras variáveis explicativas, serão necessários para uma maior compreensão sobre os entraves para o uso adequado da CSC em nosso meio.

Há mais de duas décadas os programas de saúde da criança no Brasil apóiam-se em um instrumento simples, único e progressivamente mais completo para acompanhamento da saúde infantil. Sua importância no monitoramento, vigilância e promoção da saúde infantil, seja no nível individual quanto coletivo, é inquestionável. Os resultados apresentados no presente estudo vêm somar-se a outros que o antecederam ao apontar a precária utilização dos instrumentos de acompanhamento infantil em nosso meio. Foi possível também identificar alguns fatores implicados na qualidade do preenchimento da atual CSC na amostra estudada. A análise conjunta destes fatores reforça a necessidade de estimular os profissionais da rede pública de saúde para uma abordagem integral da saúde da criança e para a utilização da CSC para o acompanhamento da saúde infantil. Reforça também a necessidade de investimentos permanentes em capacitação dos profissionais e organização dos serviços para que a contribuição da CSC para a melhoria da saúde das crianças neste País seja potencializada. Além disso, é preciso que os serviços de saúde se organizem para otimizar a ida das crianças às UBS, especialmente para a vacinação, como oportunidades de abordagem e registro dos demais aspectos de sua saúde. Foi mostrado também que a sensibilização e o envolvimento das famílias podem contribuir para o bom uso da CSC.

Vale reforçar que, apesar das mudanças epidemiológicas vividas nas últimas décadas, boa parte dos problemas de saúde da população infanto-juvenil no Brasil ainda pode ser enfrentada com uma política forte para atenção básica, construída com base nas evidências científicas e acessível a todas as crianças deste país. A CSC configura-se um instrumento atual, capaz de integrar várias dessas ações e que deve ser definitivamente incorporado às práticas de profissionais e serviços de todos os níveis assistenciais.

\section{Resumo}

A Caderneta de Saúde da Criança (CSC) é um documento imprescindivel para a promoção da saúde infantil. Foi realizado um estudo transversal com amostra aleatória simples de crianças acompanhadas no Sistema Único de Saúde (SUS), visando analisar os fatores associados à qualidade do preenchimento da CSC. Foram realizadas entrevistas e verificação direta das cadernetas de 365 crianças das nove regionais de saúde de Belo Horizonte, Minas Gerais, Brasil. Foi criado um sistema de escore para classificar as CSC quanto à qualidade do seu preenchimento e relacionála às variáveis explicativas. O odds ratio foi calculado por regressão logística. O preenchimento dos vinte itens do escore variou de 3,1\% (uso de ferruginoso) a
99,7\% (data de nascimento). Os fatores associados aos piores escores foram: crianças $>12$ meses de idade (OR $=1,77)$, mães $\leq 6$ anos de estudo $(O R=1,97)$, crianças não acompanhadas por médicos generalistas $(\mathrm{OR}=$ $3,18)$ e mães que não receberam explicações sobre a $C S C$ na maternidade $(O R=1,77)$. Os resultados apontam a precária utilização da CSC, reforçando a necessidade de investimentos em capacitação dos profissionais e organização dos serviços para que ela cumpra seu papel na promoção da saúde infantil.

Saúde da Criança; Registros de Saúde Pessoal; Atenção Primária à Saúde; Promoção da Saúde 


\section{Colaboradores}

Os autores participaram do planejamento e execução da pesquisa de campo e da redação do manuscrito.

\section{Agradecimentos}

À Secretaria Municipal de Saúde de Belo Horizonte (SMSA-BH), em especial à Dra. Sônia Lansky, e ao Núcleo de Educação em Saúde Coletiva da Faculdade de Medicina da Universidade Federal de Minas Gerais, pelo apoio institucional e financeiro recebidos, sem os quais este estudo não seria possível. Aos estudantes de medicina que participaram da coleta de dados, pelo empenho e compromisso com esta pesquisa.

\section{Referências}

1. Departamento de Ações Programáticas Estratégicas, Secretaria de Atenção à Saúde, Ministério da Saúde. Agenda de compromissos para a saúde integral da criança e redução da mortalidade infantil. http://bvsms.saude.gov.br/bvs/publica coes/agenda_compro_crianca.pdf (acessado em 09/Mar/2008).

2. Departamento de Ações Programáticas Estratégicas, Secretaria de Atenção à Saúde, Ministério da Saúde. Manual para utilização da caderneta de saúde da criança. Brasília: Ministério da Saúde; 2005.

3. Agência Brasil. Nova Caderneta de Saúde da Criança traz orientações sobre registro civil e doenças. http://www.agenciabrasil.gov.br/noticias/ 2007/02/23/materia.2007-02-23.6387327120/view (acessado em 26/Fev/2007).

4. Vieira GO, Vieira TO, Costa MC, Netto PV, CabralVA. Uso do Cartão da Criança em Feira de Santana, Bahia. Rev Bras Saúde Matern Infant 2005; 5:177-84.

5. Ratis CA, Batista Filho M. Aspectos estruturais e processuais da vigilância do crescimento de menores de cinco anos em serviços públicos de saúde do Estado de Pernambuco. Rev Bras Epidemiol 2004; 7:44-53.

6. Figueiras AC, Barros LC, Barléte KC, Faria EC, Fernandes MS, Santos JR. Uso do Cartão da Criança no município de Belém. Rev Paraense Med 2001; 15:39.

7. Santos SR, Cunha AJ, Gamba CM, Machado FG, Leal Filho JM, Moreira NL. Avaliação da assistência à saúde da mulher e da criança em localidade urbana da região Sudeste do Brasil. Rev Saúde Pública $2000 ; 34: 266-71$.
8. Goulart LMHF, Alves CRL, Viana MRA, Moulin ZS, Carmo GAA, Costa JGD, et al. Caderneta de Saúde da Criança: avaliação do preenchimento dos dados sobre gravidez, parto e recém-nascido. Rev Paul Pediatr 2008; 26:106-12.

9. Secretaria Municipal de Saúde de Belo Horizonte. Sistema Municipal de Saúde. http://portal2.pbh. gov.br/pbh/index.html?id_conteudo=3165\&id_ni vel1=-1 (acessado em 15/Jul/2008).

10. Martins LB, Costa-Paiva L, Osis MJ, Sousa MH, Pinto Neto AM, Tadini V. Conhecimento sobre métodos anticoncepcionais por estudantes adolescentes. Rev Saúde Pública 2006; 40:57-64.

11. Giugliani ER, Rocha VL, Neves JM, Polanczyk CA, Seffrin CF, Susin LO. Conhecimentos maternos em amamentação e fatores associados. J Pediatr 1995; 71:77-81

12. Greenland S. Modeling and variable selection in epidemiologic analysis. Am J Public Health 1989, 79:340-9.

13. Venâncio SI, Escuder MM, Kitoko P, Rea MF, Monteiro CA. Freqüência e determinantes do aleitamento materno em municípios do Estado de São Paulo. Rev Saúde Pública 2002; 36:313-8.

14. Caldeira AP, Oliveira RM, Rodrigues OA. Qualidade da assistência materno-infantil em diferentes modelos de atenção primária. http://www.abrasco. org.br/cienciaesaudecoletiva/artigos/artigo_int. php?id_artigo=1525 (acessado em 06/Mar/2008).

15. César JA, Mendoza-Sassi R, Horta BL, Ribeiro PR, D’Ávila AC, Santos FM, et al. Indicadores básicos de saúde infantil em área urbana no extremo sul do Brasil: estimando prevalências e avaliando diferenciais. J Pediatr 2006; 82:437-44. 
16. De Onis M, Wijnhoven TM, Onyango AW. Worldwide practices in child growth monitoring. J Pediatr 2004; 144:461-5.

17. Secretaria Municipal de Saúde de Belo Horizonte. Agenda de compromissos pela saúde integral da criança e adolescente e redução da mortalidade infantil. Belo Horizonte: Secretaria Municipal de Saúde de Belo Horizonte; 2004.

18. Figueiras ACM, Puccini RF, Silva EMK, Pedromônico MRM. Avaliação das práticas e conhecimentos de profissionais da atenção primária à saúde sobre vigilância do desenvolvimento infantil. Cad Saúde Pública 2003; 19:1691-9.

19. Vincelet C, Tabone MD, Bonnefoi MC, Chevallier B, Lemaire JP, Dommergues JP. Le carnet de santé de l'enfant est-il informative? Évaluation dans différentes structures de prévention et de soins. Arch Pediatr 2003; 10:403-9.
20. Alves CR, Alvim CG, Goulart LM, Magalhães ME, Viana MR, Moulin ZS, et al. Avaliação do conhecimento de alunos do curso de especialização em saúde da família da UFMG sobre a saúde da criança e do adolescente - 2002/2003. Rev Méd Minas Gerais 2006; 15:213-8.

21. Piccini RX, Facchini LA, Tomasi E, Thumé E, Silveira DS, Siqueira FV, et al. Efetividade da atenção pré-natal e de puericultura em unidades básicas de saúde do Sul e do Nordeste do Brasil. Rev Bras Saúde Matern Infant 2007; 7:75-82.

22. Bezerra LC, Frias PG, Vidal SA, Macedo VC, Vanderlei LC. Aleitamento materno: avaliação da implantação do programa em unidades básicas de saúde do Recife, Pernambuco (2002). Ciênc Saúde Coletiva 2007; 12:1309-17.

Recebido em 13/Mar/2008

Versão final reapresentada em 25/Ago/2008

Aprovado em 29/Set/2008 